\title{
Stabilised Updated Lagrangian SPH for Fast Solid Dynamics
}

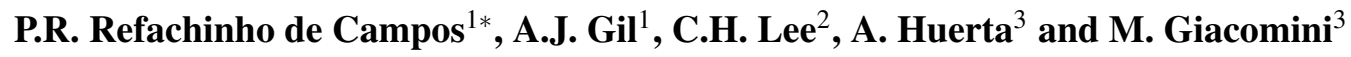 \\ ${ }^{1}$ Zienkiewicz Centre for Computational Engineering, Swansea University, \\ p.refachinhodecampos@swansea.ac.uk, a.j.gil@swansea.ac.uk
}

\author{
${ }^{2}$ Glasgow Computational Engineering Centre, University of Glasgow, chunhean.lee@ glasgow.ac.uk \\ ${ }^{3}$ Laboratori de Càlcul Numèric (LaCàN), Universitat Politècnica de Catalunya, antonio.huerta@upc.edu, \\ matteo.giacomini@upc.edu
}

\begin{abstract}
Well-established computational techniques, such as the Finite Element or Finite Volume methods, rely on the tessellation of the domain of interest into a number of interconnected non-overlapping elements or control volumes (the so-called computational mesh). Despite their enormous success from the modelling standpoint, these mesh-based methods can suffer from mesh entanglement when simulating extreme deformation processes, such as dynamic fracture. To circumvent this shortcoming, a wealth of alternative computational techniques, known as meshless or meshfree methods, emerged, where the domain is instead discretised into a set of moving particles which can interact within the region defined by a compact support. One of the earliest meshfree methodologies is the Smooth Particle Hydrodynamics (SPH), which in its classical Updated Lagrangian (displacement-based) formulation [1], can exhibit one or more of the following drawbacks: tensile instability, lack of consistency, loss of conservation, long term instability and reduced order of convergence for derived variables such as stresses and strains.

To address the above shortcomings, a new Updated Lagrangian SPH computational framework established upon a system of first order conservation laws [2] will be presented. Specifically, the usual linear momentum conservation equation will be solved in conjunction with a set of geometric conservation laws describing the motion of a continuum. Unlike previous work [2], the geometric conservation laws will be re-formulated based on a multiplicative decomposition strategy. The key feature of this formulation is the multiplicative decomposition of the deformation tensor into a known mapping and an incremental deformation. This will be achieved by considering that the original configuration will deform into the current configuration through a series of intermediate configurations. Taking advantage of this new formalism, an entropy-stable upwiding stabilisation method derived by means of Rankine Hugoniot jump conditions will be introduced. No ad-hoc algorithmic regularisation procedures are needed. Finally, and to demonstrate the robustness and applicability of the methodology, a wide spectrum of challenging problems will be presented and compared. The overall methodology will be shown to be capable of handling massive deformation problems, even in the case of shock dominated scenarios. This will open up interesting possibilities for modelling in the field of dynamic fracture, where this consideration is very relevant.
\end{abstract}

\section{REFERENCES}

[1] Vidal, Y., Bonet, J. and Huerta, A. Stabilized updated Lagrangian corrected SPH for explicit dynamics problems. Int. J. Numer. Meth. Engng. (2007) 69:2687-2710.

[2] Lee, C.H., Gil, A.J., Ghavamian, A. and Bonet, J. A Total Lagrangian upwind Smooth Particle Hydronamics algorithm for large strain explicit solid dynamics. Comput. Methods Appl. Mech. Engrg. (2019) 344:209-250. 